\title{
MODELACIÓN DE LA DINÁMICA PLAGA-PARASITOIDE-BOSQUE MEDIANTE AUTÓMATAS CELULARES.
}

Valentin Barros', Horacio Gilabert ${ }^{2}$

\section{RESUMEN}

Se presenta un modelo que simula la dinámica de una población plaga genérica basada en un sistema de autómatas celulares que permite predecir la densidad de la plaga en el tiempo y espacio. Se asumió que el crecimiento de la plaga se encuentra limitado a la disponibilidad de recurso alimenticio disponible en el bosque y a la presencia de parasitoides, que fue cuantificado mediante el modelo dinámico hospedero-parasitoide de Beddington et al. (1978), el cual es modificado incorporándole la espacialidad y dispersión de ambas poblaciones. La aplicación del modelo propuesto se realizó a un caso teórico, simulándose dos escenarios; en el primero de ellos la plaga crece y se dispersa en ausencia de parasitoides, mientras que en el segundo escenario se libera individuos de parasitoides cuando la densidad de la plaga alcanza una densidad umbral. Si bien al finalizar la simulación no se llegó a un equilibro para ambas poblaciones, los resultados demostraron que la liberación de parasitoides reduce la densidad y tasa de dispersión de la plaga, invadiendo una menor área en comparación con el primer escenario.

Palabras claves: Control biológico, parastoide, dispersión.

\section{SUMMARY}

This work presents a model that simulates the population dynamics of a generic pest by using a cellular automaton that allows the prediction of pest density on time and space. A basic assumption is made regarding the dependence and limitation of pest growth on food availability in the forest and the presence of parasitoids quantified through a dynamic host-parasitoid model proposed by Beddington et al. (1978) that is modified incorporating spatial dispersion on both populations. The model is applied into a theoretical case by simulating 2 scenarios. in the first instance the pest grows and disperses without interaction with parasitoids while in the second once a threshold pest population level is achieved, parasitoids are "released". Even though at the end of the simulation equilibrium has not been achieved, results show that releasing parasitoids decreases pest density and dispersion rate, invading a smaller area as compared with the non-release scenario.

Key words: Biological control, parasitoid, dispersal.

\footnotetext{
' Ingeniero Forestal de la Pontificia Universidad Católica de Chile. E-mail: vebarros@uc.cl

${ }^{2}$ Departamento de Ciencias Forestales. Pontificia Universidad Católica de Chile. E-mail: hgilab@uc.cl
} 


\section{INTRODUCCIÓN}

En Chile las amenazas de plagas forestales son altamente probables debido a la estructura de monocultivo de las plantaciones presentes en el pais y al incremento del comercio internacional que favorece la introducción de plagas y enfermedades forestales, poniendo en riesgo el valor económico, social y ambiental de los bosques (Baldini et al., 2005).

Esta realidad ha llevado a diferentes instituciones del Estado y privadas a proteger el recurso forestal contra el ingreso de plagas y mitigar el daño causado por su presencia a través del manejo integrado de plaga, aplicando sincrónicamente una serie de actividades basado en el manejo del bosque y en el control biológico (Baldini et al., 2005). Mills y Getz (1996) señalan que el control biológico consiste en la introducción y establecimiento de uno o varios enemigos naturales de la región de origen de la plaga con el objetivo de suprimir la abundancia de la población plaga en una región determinada a un nivel en el cual no cause más daño económico.

Los parasitoides son una clase de insectos que viven parasitando durante su etapa larval sobre un individuo de la población anfitrión (plaga) causándole la muerte y son los organismos que con más frecuencia son utilizados en el control biológico debido a dos caracteristicas principales. En primer lugar, reducen la densidad regional de la población anfitrión a un nivel inferior a la abundancia inicial después del establecimiento de individuos de parasitoides y en segundo lugar, una vez reducida la densidad de la población anfitriona, su densidad regional es mantenida en densidades bajas a menos que la interacción sea interrumpida (Mills y Getz, 1996).

Los objetivos de las medidas de control pueden ser muy variados, sin embargo, cualquiera que sea el objetivo de la medida de control, se requiere la predicción del cambio demográfico de la plaga en el tiempo y espacio (Zhou y Liebhold, 1995; Sharov, 1996).

La temporalidad y espacialidad en la dinámica de plagas toma mayor relevancia al considerar las etapas involucradas en la invasión y propagación de plagas (O'Neill et al., 1992); (i) introducción inicial, (ii) establecimiento en el nuevo hábitat y (iii) propagación en el nuevo ambiente colonizado. Estas etapas se logran a través del crecimiento y dispersión de la plaga, pudiendo alcanzar rodales no infectados en los periodos siguientes de su introducción inicial.

En los últimos años la modelación y simulación han sido aplicadas como una herramienta útil en la predicción de dinámica de poblaciones, permitiendo predecir los cambios en la densidad y dispersión de plagas, destacándose los modelos basados en autómatas celulares por su simplicidad matemática al no utilizar ecuaciones diferenciales para simular fenómenos biológicos complejos (Molofsky y Bever, 2004).

La dispersión de plagas en el espacio y tiempo ha sido modelada por diferentes autores, como Zhou y Liebhold (1995) quienes utilizan autómatas celulares para proyectar áreas afectadas por Lymantria dispar, considerando el estado de defoliación del área por la cual se dispersa la plaga, pero no consideran la presencia de controladores biológicos. A su vez, Mills y Getz (1996) revisan y discuten diferentes modelos dinámico plaga-parasitoide, pero no 
consideran la intrarelación entre la plaga y el bosque.

Este trabajo tiene por objetivo desarrollar un modelo de simulación espacial y temporal basado en autómatas celulares que permita predecir el crecimiento y dispersión de una población plaga genérica que se dispersa sobre un área forestal susceptible a ser invadida, considerando la dinámica poblacional de la plaga, sus interacciones con el bosque y una población de controladores biológicos conformada por individuos de parasitoides que limitan el crecimiento y dispersión de la población plaga. La implementación del modelo propuesto es un caso teórico y la interpretación de los resultados de dos escenarios simulados son comparados y discutidos.

\section{MATERIAL Y MÉTODOS}

\section{Autómatas Celulares}

Los autómatas celulares (ACs) son sistemas dinámicos donde el tiempo y el espacio son discretos y están compuestos por un conjunto de celdas elementales homogéneas ordenadas en una grilla regular espacial de $n$-dimensiones. Cada celda representa un lugar espacial susceptible de encontrarse en cualquiera de los estados finitos que componen el sistema. Este estado puede ser cambiado o alterado de un periodo al siguiente a través de ciertas reglas de evolución de estado, mediante un conjunto de reglas locales que involucran el estado de la celda y de sus celdas cercanas (vecindad), suponiendo que el tiempo se incrementa en forma discreta (Weimar, 1997). La actualización de estos estados se hace en paralelo siguiendo una regla local que considera sólo la vecindad de cada celda.

Los ACs son considerados como una herramienta útil para modelar sistemas complejos y extensos que han cobrado importancia en los sistemas dinámicos discretos debido a su simplicidad matemática y a la tecnologia computacional alcanzada en la actualidad. La idea central que gobierna la modelación mediante ACs es de reglas simples, pero capaces de modelar un comportamiento global complejo como consecuencia de las interacciones entre ellas (Weimar, 1997; llachinski, 2001).

Los ACs pueden definirse a través de cinco elementos básicos: celda, estado, tiempo, vecindad y reglas de transición (Karafyllidis y Thanailakis, 1997; Weimar, 1997). La celda es el elemento básico de los ACs, simboliza un lugar del espacio a modelar y guarda un estado. El estado es un número o una propiedad de la celda, que puede representar la densidad de una población en esa celda o el tipo de cobertura vegetal que posee. El estado de las celdas cambia de un instante a otro de acuerdo a un conjunto de reglas de transición comunes a todas las celdas.

El tiempo es discreto y sirve como indice en la configuración sucesiva de las simulaciones. Es tambièn homogéneo y por lo tanto esas evoluciones toman lugar de la misma forma, independiente del lugar donde ocurran.

La vecindad puede ser definida de diferentes formas, pero siempre cada celda del autómata celular se relaciona con las inmediatamente adyacentes. Una de las vecindades más 
comunes en una grilla de 2-dimensiones es la de Moore de radio uno, que considera las celdas adyacentes y diagonales, es decir las ocho celdas circundantes.

Las reglas de transición son funciones locales ya que ellas definen el estado de la celda en el tiempo $t+1$ basada solamente en su propio estado y vecindad en el instante $t$. También son funciones uniformes ya que las reglas son las mismas en cualquier lugar.

\section{Área a Modelar}

El patrimonio forestal afectado y susceptible a ser invadido por la plaga es dividido en una matriz de celdas cuadradas, donde cada celda representa a una celda de un sistema de $\mathrm{AC}$ bidimensional con vecindad de Moore de radio uno que cuantifica y determina a las poblaciones de plaga y parasitoides por la combinación de los procesos de crecimiento y dispersión de ambas poblaciones. EI AC esta compuesto por tres mallas sobrepuestas. La primera malla contiene las caracteristicas del bosque, que es representado mediante la capacidad de carga, mientras que la segunda y tercera malla contiene las densidades de la plaga y parasitoides respectivamente. De esta manera, la capacidad de carga y las densidades de plaga y parasitoides en un lugar del patrimonio forestal están dadas por los estados de las celdas correspondientes a ese lugar.

La secuencia de eventos en cada celda y periodo es como sigue: La población plaga $N_{t}$ en la celda $i$ en el periodo $t$ se reproduce, pero debido a la existencia de parasitoides, individuos de la población plaga son parasitados y mueren, proceso que es cuantificado mediante el modelo discreto de hospedero-parasitoide Beddington et al. (1978) (citado por Mills y Getz, 1996; Brassil y Abrams, 2004). Los individuos sobrevivientes compiten por los recursos de la celda, motivando a una proporción de la población plaga a dispersarse a las celdas vecinas, mientras que desde celdas vecinas llegan nuevos individuos. Los individuos de la población de parasitoides una vez que han parasitado, compiten entre sí permitiendo la dispersión hacia las celdas vecinas, mientras que otros individuos de parasitoides inmigran a la celda.

\section{Crecimiento de las Poblaciones de Plaga y Parasitoides}

Se asume en las condiciones iniciales de la simulación $\left(t_{0}\right)$ la existencia de individuos de plaga en algunas celdas y que la densidad de parasitoides es cero en todas las celdas, pudiendo ser liberado individuos de parasitoides desde el periodo siguiente $(t$,$) hasta el final del$ horizonte de simulación $T$.

El crecimiento de ambas poblaciones es determinado usando el modelo discreto de dinámica hospedero-parasitoide de Beddington et al. (1978) (citado por Mills y Getz, 1996; Brassil y Abrams, 2004), el cual es modificado incorporándole la ubicación espacial de las poblaciones y la liberación de parasitoides mediante las siguientes ecuaciones:

$$
\begin{gathered}
N_{i(t+1)}=N_{1} \cdot \exp \left\{r\left(1-N_{1} / K_{t}\right)\right\} \cdot \exp (-a \cdot P) \\
P_{i(t+1)}=N_{1} \cdot\left(1-\exp \left(-a \cdot P_{t}\right)+X_{i(t+1)}[2]\right.
\end{gathered}
$$




\section{Donde:}

$N_{\text {y }}$ y son las densidades de plaga y parasitoides en la celda $i$ del AC en el periodo $t$ respectivamente

$r$ es la tasa de incremento de la población plaga

$K$ es la capacidad de carga de la celda $i$ en el periodo $t$ para la población de plaga

a es el área de descubrimiento ó la proporción de ambiente de la plaga que puede ser cubierta por individuo de parasitoides

$X \quad$ es la cantidad de parasitoides liberado en la celda $i$ en el periodo $t+l$.

El crecimiento de la población plaga se encuentra limitado a la disponibilidad de follaje existente en el periodo $t$ de cada celda, el cual se puede incorporar en la capacidad de carga (Ludwig et al., 1978). Asi, aquellas celdas en las que exista una mayor disponibilidad follaje podrán hospedar una mayor cantidad de individuos de plaga, permitiendo que la plaga se desarrolle primero en las celdas con soporte alimenticio para posteriormente dispersarse a otras celdas que tengan un follaje aún no consumido.

La capacidad de carga queda definido a partir del área foliar de cada celda. Para esto se asume que cada individuo de la población plaga necesita un área de follaje para su sobrevivencia durante un periodo. La capacidad de sitio para la celda $i$ para el periodo $t$ queda definido por:

$$
K_{u}=\frac{f_{\|} \cdot A F_{u}}{D}
$$

Donde:

$A F_{\text {si }}$ corresponde al área foliar de la celda $i$ en el periodo $t$

$D$ es la tasa de consumo de área de follaje por individuo de la población plaga durante un periodo $f_{t}$ es la proporción de área foliar que presenta las caracteristicas necesarias para ser utilizada como recurso alimenticio por la plaga en la celda $i$ en el periodo $t$.

Si una celda ha sufrido de la invasión de individuos de plaga la ecuación [3] es modificada para cuantificar la disminución de la capacidad de carga como consecuencia de la defoliación en los periodos anteriores, la cual es estimada a partir del coeficiente $D$ y se asume que el bosque es capaz de recuperarse de la defoliación del periodo anterior en un porcentaje $R$. El factor de recuperación $R_{\hbar}$ es la capacidad que posee el bosque de recuperar el follaje perdido en $t-1$ en la celda $i$, el cual es expresado en término de porcentaje de la defoliación ocurrido en el periodo anterior y que es recuperado en el periodo siguiente $(t)$.

La recuperación de la defoliación del período anterior es $D \cdot N_{(i t \cdot 1)} \cdot R_{t !}$ y ocurre en el periodo $t$. Debido a que la defoliación provoca una disminución del follaje y la tasa de recuperación recupera un porcentaje del follaje desfoliado, la factorización de ambos términos es $D \cdot N_{i(t-1)} ;\left(R_{t}-1\right)$ y toma valor negativo debido a que el porcentaje de recuperación es menor o igual a uno. De esta manera la capacidad de carga en cualquier celda y periodo, independiente de si ha sido infectada por plaga en periodos anteriores, queda establecida por la expresión: 


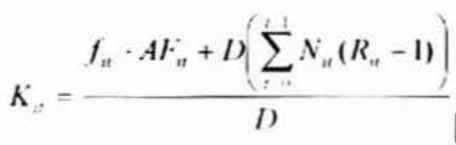

Debido a que el porcentaje de recuperación de la defoliación ocurre con un periodo de retardo, la sumatoria que cuantifica la disminución del área foliar es de $t=0$ a $t=t-1$ y da cuenta de la disminución de capacidad de carga respecto a una celda que no ha sido infectada por plaga.

\section{Dispersión de las Poblaciones}

El número de individuos de plaga que llega a la celda $i$ desde el conjunto de celdas pertenecientes a su vecindad es estimado mediante la probabilidad de dispersión $m n_{\lambda t^{\prime}}$ donde $\lambda$ son las celdas pertenecientes a la vecindad de la celda $i$. La probabilidad de dispersión hacia la celda $i$ es estimada en dos etapas. Primero se estima la probabilidad de que la población plaga abandone la celda $\lambda$ debido a la competencia intraespecifica en esa celda, y luego se calcula el porcentaje de la población que deja la celda $\lambda$ y que se dispersa a la celda $i$.

La probabilidad de que la población plaga abandone una celda es calculada asumiendo una estrategia de dispersión dependiente de densidad, para lo cual se utiliza un modelo de dispersión umbral. Este modelo de dispersión asume que la probabilidad de dispersión $\left(q_{,}\right)$se mantiene constante a medida que aumenta la densidad hasta que se llega a una densidad umbral $\left(\mathrm{N}_{i,}^{*}\right)$ en la cual la competencia intraespecifica se intensifica y la probabilidad de dispersión comienza a aumentar en forma lineal, hasta llegar a una probabilidad máxima de dispersión (Johst y Brandl, 1997).

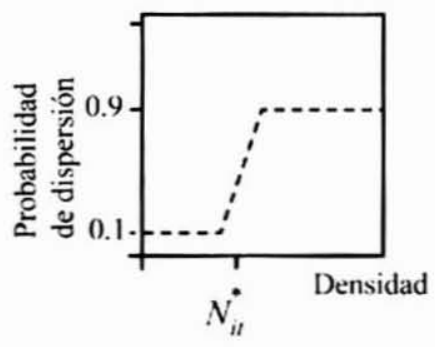

Figura $\mathrm{N}^{\circ} 1$

DISPERSIÓN UMBRAL

Luego de estimar la probabilidad $q_{i,}$ a partir de la Figura $N^{\circ} 1$, se calcula una nueva probabilidad que indica el porcentaje de la población plaga que emigra de la celda $\lambda$ para dispersarse a la celda $i\left(p q_{i, t}\right)$, las que son calculadas a partir de la capacidad de carga $\left(K_{\text {, }}\right)$ de la 
vecindad de cada una de las celdas ;.. De esta manera, la capacidad de carga es utilizada como un indicador de preferencia para la dispersión, permitiendo a la población plaga dispersarse en un mayor porcentaje a las celdas que tengan más recurso alimenticio, el cual se encuentra incorporado en la capacidad de carga de cada celda.

Asi, las probabilidades de dispersión de la población plaga hacia la celda $i$ desde su vecindad durante el periodo $t$ queda definida como

$$
\mathrm{mn}_{i . i t}=q_{\lambda . t} \bullet p q_{i, i t}[5]
$$

Donde:

$m n_{, 1}$ es la probabilidad de que un individuo de la celda $\lambda$ se disperse a la celda $i$ en el período $t$. La probabilidad $m n_{, \prime \prime}$ corresponde a la proporción de la población plaga de la celda $i$ que se dispersa a la celda $i$. Cuando $i=i$

La probabilidad $m n_{, \prime \prime}$ indica la proporción de individuos de plaga que no se dispersa y permanece en la celda $i$.

Respecto a las probabilidades de dispersión para la población de parasitoides ( $m p$, ), estas son calculadas a partir de las densidades de plaga de su vecindad, que es utilizado como un indicador de atracción para los individuos de parasitoides. De esta manera, los individuos de parasitoides se dispersarán mayormente a celdas vecinas que tengan una mayor densidad de plaga.

Luego de estimar las probabilidades de dispersión de ambas poblaciones, las densidades de plaga y parasitoides en cada celda y en cualquier periodo dadas las condiciones iniciales, es determinada por el incremento de la población dado por las expresiones [1] y [2] aplicada a cada una de las celdas con la población del periodo anterior y la dispersión desde las celdas de la vecindad hacia la celda $i$ entre periodos de tiempo. La liberación de parasitoides $X_{i t+\ldots i}$ que reducen la densidad de plaga. es realizada en el periodo posterior $(t+l)$ en aquellas celdas donde la plaga alcance un porcentaje $\alpha$ de la capacidad de carga en el periodo $t\left(\alpha \cdot K_{t \prime}\right)$.

\section{Aplicación del Modelo Propuesto}

Para la aplicación teórica del modelo desarrollado, se trabajó sobre un área forestal susceptible a ser invadida por la población plaga y con valores de los diferentes parámetros requerido por el modelo $\sin$ la referencia a una plaga real o a una posición geográfica especifica. Se simuló dos escenarios; en el primero de ellos la plaga creció y se dispersó en ausencia de parasitoides, mientras que en el segundo escenario se liberaron individuos de parasitoides.

El área espacial a modelar fue dividida en una red de celdas de $30 \times 30$ donde cada celda posee una superficie de una hectárea. El área por el cual se propaga la plaga se encuentra conformada por seis rodales, los cuales son representados por un conjunto de celdas, permitiendo tener un ambiente heterogéneo en el cual la plaga crece y se dispersa a distintas 
tasas. El horizonte de simulación consistió en diecinueve periodos. La densidad de plaga al inicio de la simulación fue de 25 individuos para doce celdas (Figura $\mathrm{N}^{\circ} 2$ ).

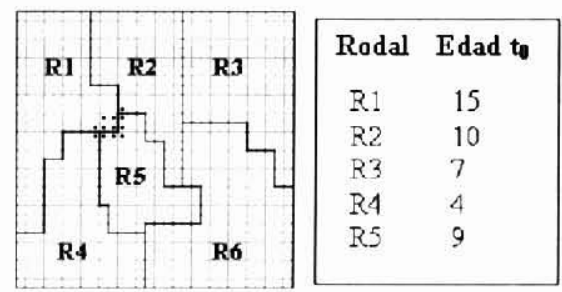

Figura $\mathrm{N}^{\circ} 2$

DISPOSICIÓN ESPACIAL DE RODALES Y CELDAS INFECTADAS EN $\boldsymbol{t}_{0}$.

Los parámetros requeridos por el modelo para la proyección de la dinámica de la plaga y parasitoides se presentan en el Cuadro $\mathrm{N}^{\circ} 1$.

\section{Cuadro $\mathrm{N}^{\circ} 1$}

VALORES DE LOS PARÁMETRO UTILIZADOS EN LAS SIMULACIONES

\begin{tabular}{|l|c|c|l|c|c|}
\hline \multicolumn{1}{|c|}{ Parámetro } & Símbolo & Valor & \multicolumn{1}{|c|}{ Parámetro } & Simbolo & Valor \\
\hline Tasa de incremento plaga & $r$ & 1,2 & Parasitoides liberado & $X_{(i++1)}$ & 20 \\
\hline Tasa consumo de follaje & $D$ & 1 & Área descubrimiento parasitoide & $a$ & 0,02 \\
\hline Nivel liberación parasitoides & $a$ & 0,15 & Población plaga umbral & $N_{\lambda, t}^{*}$ & 0,2 \\
\hline
\end{tabular}

La capacidad de carga para la población plaga de cada celda fue calculada mediante la ecuación [4]. Para ello, los valores de las área foliares se estimaron utilizando el modelo propuesto por Turner et al. (2000), quienes relacionan el diámetro de un árbol para estimar su área foliar. La proporción de follaje que puede ser consumido por la población plaga se estableció de manera tal que las celdas que poseen diámetros mayores tengan una mayor disponibilidad de follaje. Para el crecimiento del bosque se utilizó el programa Radiata Plus. La edad de rotación fue fijada a los 30 años, por lo cual el rodal 1 es cosechado en el periodo 15 y entre los periodos 16 y 18 se asumió un área foliar igual a un metro cuadrado para el conjunto de celdas que conforman el rodal 1.

\section{RESULTADOS}

La población plaga en el escenario 1 (sin liberación de parasitoides) llegó a una densidad de 3.021.695 individuos al final del horizonte de simulación, mientras que en el escenario 2 la población plaga presentó dos peak de alta densidad, siendo el segundo de ellos de mayor densidad con un total en ese periodo de 179 mil individuos. La menor densidad de plaga en el 
escenario 2 se debió a la liberación de 2.220 individuos de parasitoides, que fueron liberados a partir del cuarto periodo, las que continuaron con una mayor frecuencia en los periodos posteriores.

Respecto a la dispersión de la población plaga, esta se inició a partir del primer periodo y ambos escenarios se mantuvieron iguales hasta el sexto periodo, donde la dispersión de la plaga comenzó a diferenciarse debido a la liberación de individuos de parasitoides en el escenario 2.

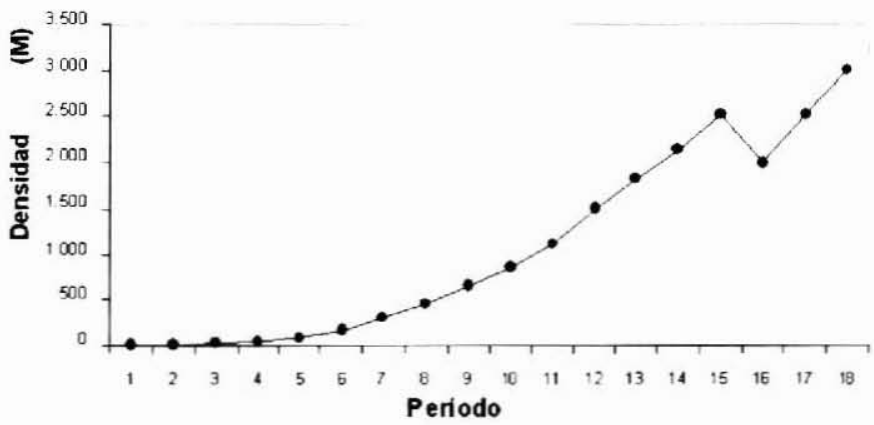

\section{- Densidad Plaga}

Figura $\mathrm{N}^{\circ} 3$

DENSIDAD DE LA POBLACIÓN PLAGA (MILES) PARA ESCENARIO 1

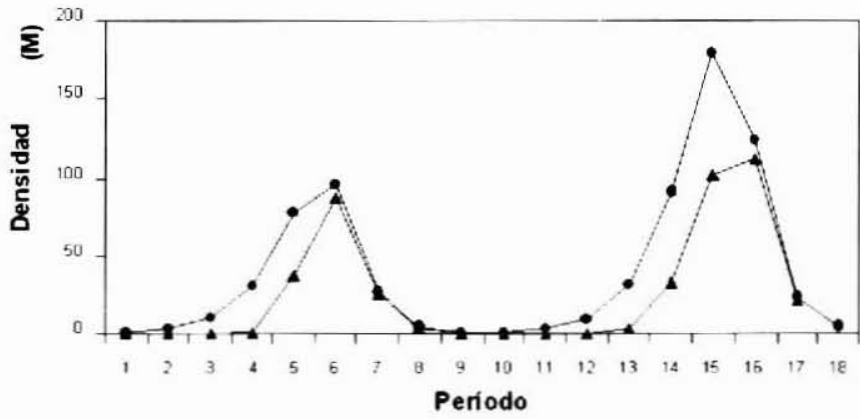

- Densidad Plaga $\rightarrow$ Densidad de Parasitoides

Figura $\mathbf{N}^{\circ} 4$

DENSIDADES DE LAS POBLACIONES DE PLAGA Y PARASITOIDE (MILES) PARA ESCENARIO 2

La menor densidad de plaga en el escenario 2 motivó una disminución en las probabilidades de dispersión debido a un menor nivel de competencia existente en las celdas infectadas. Es asi como el área infectada en el escenario 2 sólo alcanzó un $44 \%$ de las celdas 
infectadas en el escenario 1 al finalizar la simulación (Figura $\mathrm{N}^{\circ} 5$ ).

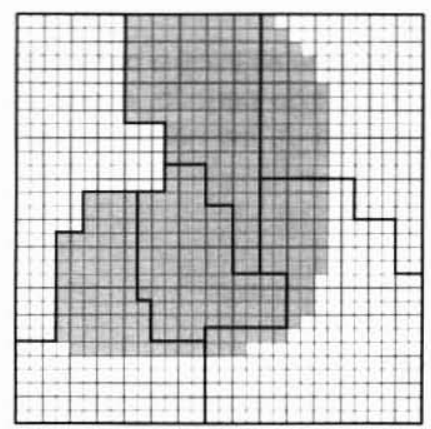

Escenario 1

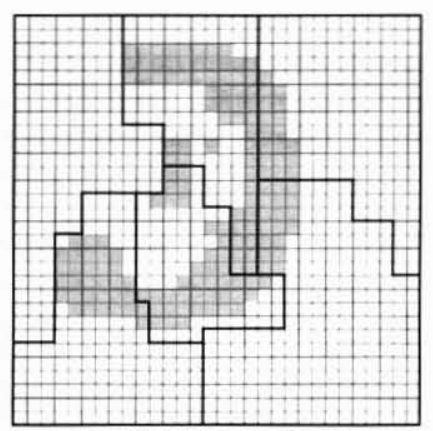

Escenanio 2

Figura $\mathrm{N}^{\circ} 5$

ÁREA INVADIDA POR LA POBLACIÓN PLAGA EN EL PERIODO 18

La dinámica de la dispersión de la plaga muestra que los individuos tienen una preferencia para dispersarse hacia el área Este del área modelada, lugar donde se encuentra el rodal 1. Este patrón de dispersión causó que las densidades de la plaga en ese rodal fueran mayores y el área infectada perdiera su simetria inicial y haya tomando una forma ovalada. Esta situación se debió a que el rodal 1 es el de mayor edad, y presentó una mayor disponibilidad de recurso alimenticio, que es una función de su diámetro. Sin embargo, la cosecha del rodal 1 en el periodo 15 provocó la extinción de los individuos de plaga presente en esas celdas, situación que se refleja en la disminución en la densidad total de la plaga.

La mayor concentración de individuos de plaga se mostró en las celdas infectadas al inicio de la simulación y en sus celdas vecinas, mientras que las celdas limites del área infectada presentaron una densidad inferior. Esto se explica por el hecho de que antes de dispersarse, la plaga coloniza la celda aumentando su densidad, originando que un pequeño número de individuos de plaga se dispersen a las celdas vecinas que delimitan el área afectada por plaga.

\section{DISCUSIÓN}

La menor densidad de plaga en el escenario 2 revela que la dinámica de la población plaga se encuentra dirigida por los procesos de su crecimiento poblacional y disminución poblacional por mortalidad provocados por los individuos de parasitoides. Seguido a la disminución de la plaga, y con un periodo de retardo, la población de parasitoides también disminuye, reflejando que la existencia de individuos de parasitoides depende de la densidad de su hospedero.

La dinámica de dispersión de la plaga muestra que los individuos tienen una preferencia para dispersarse hacia el área Este del área modelada. Esta preferencia de la plaga para 
dispersarse preferentemente hacia un sector del área demuestra que la heterogeneidad espacial y temporal del bosque actúa como un factor relevante en la dinámica demográfica y en la propagación hacia las áreas que presentan las condiciones más atractivas para la población plaga.

La extinción de la plaga en el rodal 1 debido a su cosecha muestra que el modelo es capaz de predecir el comportamiento de los individuos de la población plaga respecto de su densidad y tasas de dispersión, al modificarse la capacidad de carga de las celdas producto de la cosecha del rodal 1. En este sentido, y basado en que en la realidad los bosques son sometidos a diversas intervenciones silviculturales, algunas de ellas tendiente a controlar plagas o disminuir la susceptibilidad de bosques a ser infectados por plaga (Cadahia, 1980 citado por Baldini et al., 2005), el modelo desarrollado es capaz de incorporar las alteraciones producida por el manejo silvicultural, modificando la capacidad de carga y las probabilidades de dispersión de la plaga.

La principal limitación del proceso de dispersión es que sólo permite la dispersión a las celdas vecinas, impidiendo alcanzar una mayor distancia de propagación. Sin embargo, para las especies que poseen una alta capacidad de dispersión, las reglas de transición que regulan la dispersión pueden ser insuficientes para cubrir la extensión espacial de esas plagas, por lo cual se proponen las siguientes alternativas: (i) utilizar una vecindad de Moore de radio mayor a uno, permitiendo abarcar una mayor distancia de dispersión en un periodo, y (ii) aumentar las superficies de las celdas, obteniéndose de esta manera que la distancia entre dos celdas vecinas sea mayor.

Las densidades de ambas poblaciones no llegan a un equilibrio al finalizar la simulación. al cual se debería llegar a menos que la interacción entre ambas poblaciones sea interrumpida (Mills y Getz, 1996). Esta situación se puede explicar en que la interacción entre hospederoparasitoides es semejante a la dinámica entre depredador y presa. Nebel y Wright (1999) señalan que en términos de equilibrio poblacional, los organismos parasitarios actúan del mismo modo que los depredadores. Cuando la densidad del hospedero aumenta, los parasitoides tienen pocas dificultades en encontrar nuevos hospederos, y los indices de parasitismos crecen y causan muertes en los individuos de hospedero. Al contrario, cuando la densidad del hospedero es baja, se impide el parasitismo y hay un gran número de parasitoides afectados, condición que permite que la población hospedera se recupere.

Para validar el modelo desarrollado para verificar su aplicación práctica, es indispensable que la relación entre la plaga y su controlador biológico sea compatible con el modelo dinámico hospedero-parasitoide de Beddington et al. (1978). Este modelo de hospedero-parasitoide incorpora la dependencia a la densidad de anfitrión para agregarle estabilidad al modelo original de Nicholson y Bailey (Mills y Getz, 1996). Sin embargo, en el ejemplo teórico presentado no se llega a un equilibrio estable durante el periodo de simulación. Esta situación lleva a seguir investigando la dinámica hospedero-parasitoide que permitan predecir las densidades de ambas poblaciones y si estas llegan a un equilibrio estable.

Si bien los individuos de parasitoides reducen la densidad y dispersión de la población plaga, el modelo no entrega una asignación óptima en la liberación de parasitoides al no 
realizar un análisis de costo-beneficio, impidiendo asegurar un equilibrio óptimo entre el daño ocasionado por la plaga y los costos asociados a la medida de control (Zhou y Liebhold, 1995; Sharov, 1996).

\section{CONCLUSIONES}

Los resultados muestran que un sistema de simulación basado en autómatas celulares es capaz de simular y predecir el comportamiento de un sistema ecológico complejo, utilizando un pequeño número de reglas simples. Además, demuestra que es posible predecir el crecimiento y dispersión de una plaga en presencia o ausencia de controladores biológicos.

Los modelos demográficos de plagas no pueden ser considerados si ellos no reflejan los procesos de crecimiento y dispersión de poblaciones, debido que su dinámica se ve fuertemente relacionada e influenciada espacial y temporalmente por los dos procesos señalados.

La densidad y dispersión de la plaga en el escenario 2 se ve afectadas por la liberación de parasitoides. Es asi como el área invadida sólo alcanza el $44 \%$ de las celdas infectadas respecto al escenario 1. Este hecho demuestra que la liberación de controladores biológicos disminuye la probabilidad de que la plaga llegue a áreas no infectadas.

La heterogeneidad espacial del hábitat influye en la dispersión de la plaga. Este acontecimiento demuestra que las diferentes características del bosque son un factor relevante en la dinámica demográfica de la plaga hacia aquellas áreas que presentan las condiciones apropiadas.

La liberación de parasitoides disminuye la densidad de la plaga durante todo el transcurso de la simulación. Sin embargo, ambas poblaciones presentan un comportamiento fluctuante en sus densidades, por lo cual el objetivo que conlleva la liberación de controladores biológicos. que es mantener a la plaga a una baja densidad, no se puede asegurar por los resultados obtenidos. Esta situación lleva a seguir investigando la dinámica hospedero-parasitoide, que permita predecir las densidades de ambas poblaciones y si estas llegan a un equilibrio estable y no fluctuante como en los resultados presentados.

\section{REFERENCIAS}

Baldini, A., G. Cogollor, A. Sartori y J. Aguayo, 2005. Control biológico de plagas forestales de importancia económica en Chile. CONAF. Chile. 205p.

Brassil, C. and P. Abrams, 2004. The prevalence of asymmetrical indirect effects in two-host-oneparasitoid systems. Theoretical Population Biology 66(1): 71-82.

Ilachinski, A., 2001. Cellular automata: a discrete universe. World Scientific. 808p. 
Johst, K. and R. Brandl, 1997. Evolution of dispersal: the importance of the temporal order of reproduction and dispersal. The Royal Society 264: 23-30.

Karafyllidis, I. and A. Thanailakis, 1997. A model for predicting forest fire spreading using cellular automata. Ecological Modelling 99: 87-97

Ludwig, D., D. JONES and C. HOLLING, 1978. Qualitative analysis of insect outbreak systems: the Spruce Budworm and forest. Journal of Animal Ecology 47. 315-332.

Mills, N. and W. Getz, 1996. Modelling the biological control of insect pests: a review of host-parasitoid models. Ecological Modelling 96: 121-143.

Molofsky, J. and J. Bever, 2004. A new kind of ecology? BioScience 54(5): 440-446.

Nebel, B. and Wright, 1999. Environmental science: the way world work. Sixth Edition. Prentice Hall. E.U.A. 720p.

O'neill, R., R. Gardner, M. Turner and W. Romme, 1992. Epidemiology theory and disturbance spread on landscapes. Landscape Ecology 7(1): 19-26.

Sharov, A., 1996. Modelling forest insect dynamics. Caring for the forest: research in a changing world. Congress Report, Vol. II: 293-303. IUFRO XX World Congress, Tampere, Finlandia

Turner, D., S. Acker, J. Means and S. Garman, 2000. Assessing alternative allometric algorithms for estimating leaf area of Douglas-fir trees and stands. Forest Ecology and Management 126(1): 61-76.

Weimar, J., 1997. Simulation with cellular automata. Logos Verlag Berlin. 199p.

Zhou, G. and A. Liebhold, 1995. Forecasting the spatial dynamics of gypsy moth outbreak using cellular transition models. Landscape Ecology 10(3): 177-189. 